\title{
AN ASSOCIATION BETWEEN SEASONAL FLUCTUATION 'CYCLING' OF 25(OH)D AND INCREASED BONE RESORPTION BUT NOT BMD OR BMC IN UK SOUTH ASIAN AND CAUCASIAN WOMEN LIVING AT 51ON
}

A. L. Darling 1,*, F. Gossiel 2, R. Hannon 2, D. J. Skene 3, K. H. Hart 1, J. L. Berry 4, R. Eastell 2, S. A. Lanham-New 1

1Division of Nutritional Sciences, University of Surrey, Guildford, 2Department of Human Metabolism, University of

Sheffield, Sheffield, ${ }_{3}$ Chronobiology, University of Surrey, Guildford, ${ }_{4}$ Vitamin D Research Group, School of Biomedicine,

University of Manchester, Manchester, United Kingdom

Abstract: It has been hypothesised that the $U$ shaped association between 25(OH)D and some health outcomes may be due to large seasonal fluctuations of $25(\mathrm{OH}) \mathrm{D}_{1}$. It is unknown whether such fluctuation of 25(OH)D ('cycling') influences bone health. This is an important issue, because if 'cycling' is detrimental for bone, then winter only rather than year round vitamin D supplementation may be useful for bone health to 'blunt' the rhythm. In the D-FINES study, $n=373$ women (South Asian/Caucasian) had repeated measurements in four seasons for serum 25(OH)D and PTH, as well as a DXA scan in autumn and spring. Serum C-telopeptide (SCTX) was also measured in a random subset $(n=66)$. Cosinor regression analysis was used to identify individuals showing a significant rhythm $(p<0.10)$ ('cyclers') and those not showing a significant seasonal rhythm ('non-cyclers'). Potential differences in bone indices between the two groups were assessed within ethnicity. Dependent variables analysed were absolute values for autumn femoral neck and lumbar spine BMD, BMC and bone area, and absolute SCTX and SPTH in each season. Also, change in SCTX and SPTH from summer to winter and change in DXA bone indices from autumn to spring were analysed. ANCOVA was run, adjusting for summer and winter 25(OH)D status, age, socioeconomic status, physical activity, and dietary calcium. BMI was also controlled for in the analysis due to its negative correlation with seasonal change in $25(\mathrm{OH}) \mathrm{D}$. There was no statistically significant difference $(p>0.05)$ between 'cyclers' and 'non-cyclers' for any of the bone indices in either ethnic group.

However, there were trends for a higher CTX and PTH in 'cyclers' versus 'non-cyclers' in both ethnic groups in every season, but no differences for BMD or BMC (figures 1-4). This suggests tentatively that 'cycling' could be associated with changes in bone metabolism but may not translate into structural changes. In summary, there is no clear evidence here to suggest that 'cycling' is detrimental to bone health, although there are trends in PTH and CTX that warrant further investigation with a larger sample.

Reference

1 Vieth, R. (2004) Enzyme kinetics hypothesis to explain the U-shaped risk curve for prostate cancer vs. 25- hydroxyvitamin D in nordic countries. Int J Cancer 111(3): 468

\section{Image / Graph:}


Figures 1-4 Covariate* adjusted mean CTX, PTH, BMD and BMC

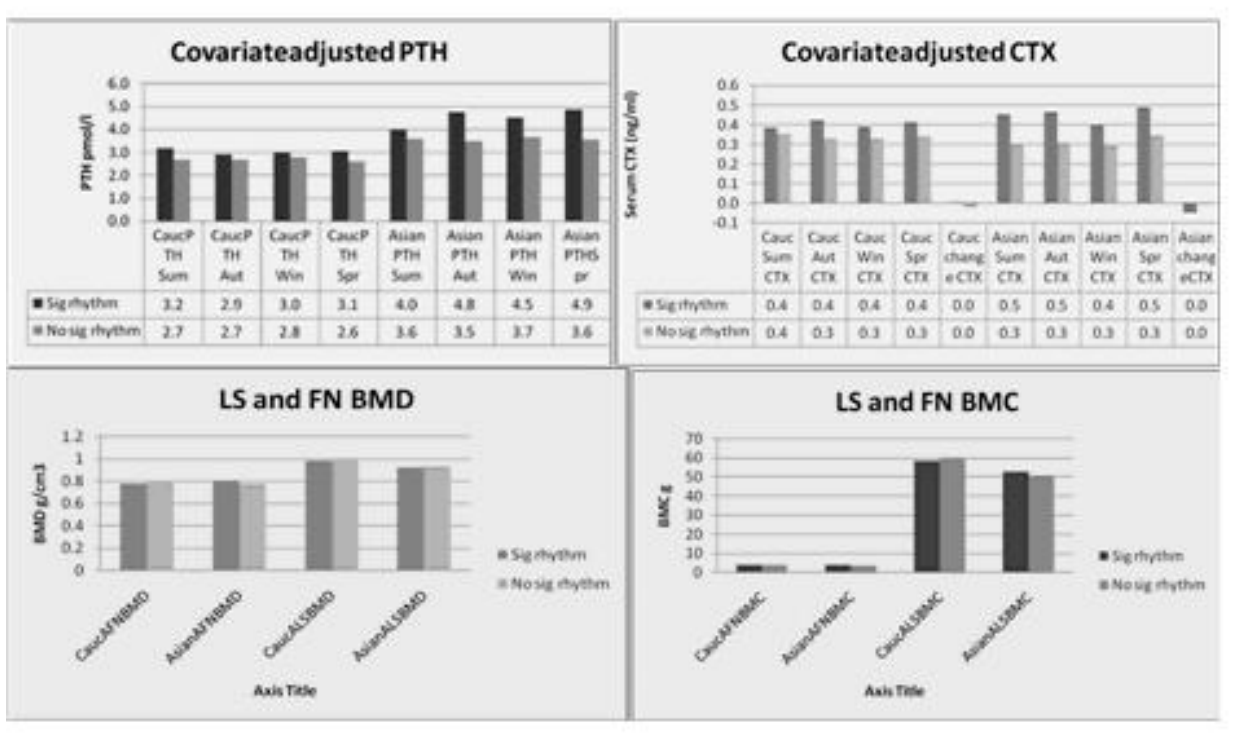

*Adjusted for summer and witer $25(\mathrm{OH}) \mathrm{D}$ status, age, $\mathrm{BMI}$, socioeconomic status, physical activity, and dietary calcium

Disclosure of Interest: None Declared 